\title{
PD-1 and PD-L1 Expression Predicts Radiosensitivity and Clinical Outcomes in Head and Neck Cancer and is Associated with HPV Infection
}

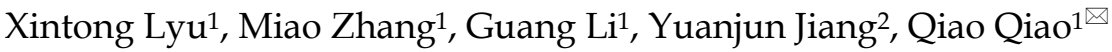 \\ 1. Department of Radiation Oncology, the First Hospital of China Medical University, Shenyang 110001, Liaoning, China \\ 2. Department of Urology, the First Hospital of China Medical University, Shenyang 110001, Liaoning, China \\ $\triangle$ Corresponding author: Qiao Qiao, Email: braveheart8063@outlook.com \\ (c) Ivyspring International Publisher. This is an open access article distributed under the terms of the Creative Commons Attribution (CC BY-NC) license \\ (https://creativecommons.org/licenses/by-nc/4.0/). See http://ivyspring.com/terms for full terms and conditions.
}

Received: 2018.05.10; Accepted: 2019.01.02; Published: 2019.01.29

\begin{abstract}
Objectives: PD-1 and PD-L1 overexpression in malignant tumors in response to radiotherapy is correlated with a poor prognosis. Human papilloma virus (HPV) infection impacts intrinsic radiosensitivity of head and neck cancers (HNCs). Herein, this study aims to determine PD-1/PD-L1 expression differences in tumors with different HPV statuses and their prognostic value in patients with different radiosensitivity gene signatures to define the characteristics of patients who will benefit from radiotherapy combined with anti-PD-1/PD-L1 therapy.

Material and methods: According to the identified gene signature related to radiosensitivity, 517 patients from the TCGA HNSCC cohort were selected and divided into the radioresistant (RR) group and radiosensitive (RS) group using a K-mean clustering algorithm. All data analyses were conducted using SPSS and GraphPad Prism.

Results: PD-Ll expression is upregulated in tumor tissue (unpaired test, $\mathrm{P}=0.0363$; paired test, $\mathrm{P}=0.0584$ ) compared with normal tissue. $\mathrm{PD}-\mathrm{Ll}$ was positively correlated with $\mathrm{PD}-1$ expression $(\mathrm{P}<0.0001)$. The HPV/p16-positive group was significantly high PD-1 expression $(P<0.0001)$. PD-L1 expression $(P=0.0005)$ and $P D-1$ expression $(P<0.0001)$ were significantly increased in the $R S$ group compared with that in the RR group. In the patients who were treated with radiotherapy, the PD-1-high group was associated with better recurrence-free survival (RFS) (HR, 0.4892; 95\% Cl, 0.2357-1.015; $P=0.023)$. Within the RR group, high PD-LI expression was associated with reduced overall survival (OS) (HR, 2.196; 95\% Cl, 1.081-4.46; P=0.0108) compared with low PD-LI expression. In the RR group, HPV/p 16-negative patients with high PD-LI expression exhibited reduced OS (HPV: HR, 2.334; 95\% Cl, 0.7828-6.961; $\mathrm{P}=0.0313$; $\mathrm{pl}$ : $\mathrm{HR}, 2.486 ; 95 \% \mathrm{Cl}, 0.8559-7.219 ; \mathrm{P}=0.0192$ ) compared with that of patients with low PD-LI expression. In the PD-L1-high group, RR patients exhibited reduced OS (HR, 0.4858; $95 \%$ $\mathrm{Cl}, 0.2136-1.105 ; \mathrm{P}=0.0189)$ and $\mathrm{RFS}(\mathrm{HR}, 0.4371 ; 95 \% \mathrm{Cl}, 0.1421-1.345 ; \mathrm{P}=0.0231)$ compared with that of RS patients.

Conclusion: Our findings demonstrated that high PD-1/PD-LI expression was strongly related to radiosensitivity, and high PD-1 expression was significantly associated with HPV/p16-positive HNCs. Patients in the radioresistant group and patients in the HPV/p 16-negative group with a radioresistant gene signature could benefit from the combination of radiotherapy and anti-PD-1/PD-L1 therapy.
\end{abstract}

Key words: PD-L1, PD-1, head and neck cancer, HPV, radiosensitivity, prognosis

\section{Introduction}

Head and neck cancer (HNC) is the ninth most common malignancy worldwide and the third most common in developing counties with high mortality rates [1]. Greater than ninety percent of HNCs are derived from squamous epithelium. Treatment of HNCs is multidisciplinary. One of the main 
treatments is radiotherapy. Currently, the development of new technologies, such as intensity-modulated radiotherapy (IMRT) and concurrent chemoradiotherapy (CCRT), have resulted in improvements in the clinical outcome of patients. However, in the past decades, the 5-year survival rate of radical radiotherapy remained at approximately fifty percent. Unfortunately, local recurrence occurs in fifty percent of patients. In this era, tumor immunotherapy has received considerable attention in the treatment of cancer. Therefore, new synergistic targets for a combination of radiotherapy with immunotherapy are urgently required.

As one of the immune escape checkpoints, programmed cell death protein 1 (PD-1), has gained considerable attention to date. Seiwert, T. Y. et al. [2] established the basis of the application of pembrolizumab, a PD-1 antibody, in the recurrence and metastasis of refractory head and neck squamous cell carcinoma (HNSCC). Currently, pembrolizumab has been approved by the Food and Drug Administration (FDA) for patients with HNSCC who have progressed, relapsed or metastasized after chemotherapy based on platinum [3]. However, the response rate of single pembrolizumab treatment was only 18 percent [2]. In addition to mediating the effects of cytotoxic activity and cytostatic activity on cancer cells, radiotherapy also exhibits immunomodulatory effects $[4,5]$. Therefore, a combination of radiotherapy and immunotherapy may improve the therapeutic response. An animal experiment demonstrated that the combination of radiation with PD-1 blockade improved local tumor control [6]. However, clinical evidence for PD-1/PD-L1 blockade combined with radiotherapy has not been reported. In addition, the relation of PD-L1/PD-1 expression and radiosensitivity has rarely been reported. One study recently demonstrated that high PD-L1 expression in head and neck cancer was associated with increased rates of response to radiotherapy, but the results failed to reach statistical significance [7]. Thus, this study aims to explore the correlation between the PD-1/PD-L1 expression and radiosensitivity in HNCs and provide a powerful theoretical basis for the clinical feasibility of anti-PD-1/PD-L1 in combination with radiotherapy.

An increasingly important risk factor for HNCs is human papilloma virus (HPV) infection. The percentage of HPV infection in HNCs is 57-72 percent. The highest incidence is noted in oropharyngeal cancer in Western countries, and HPV infection is noted in 25 percent of other oral cancers [8]. HPV-positive HNCs exhibit a better prognosis compared with HPV-negative HNCs. This suggests that the worse clinical outcomes of patients with
HPV-negative status may be related to intrinsic radiosensitivity [9]. Previous research [2] has demonstrated that the rate of pembrolizumab efficacy was significantly increased in HPV-positive HNCs. Thus, this study intends to evaluate the correlation between PD-1/PD-L1 expression and HPV and discuss the potential targets of PD-1 and PD-L1 to regulate radiosensitivity. Furthermore, we sought to provide a new evaluation index for selecting cases that will benefit from combination treatment of radiotherapy and PD-1/PD-L1 immune therapy.

In the present study, we validated a 31-gene signature that is associated with radiosensitivity in the head and neck cancer population. We then first analyzed the relationship between basal PD-1/PD-L1 expression and clinical factors, including radiosensitivity and $\mathrm{HPV} / \mathrm{p} 16$ status in a large cohort. Furthermore, we first examined the prognostic role of basal PD-1/PD-L1 expression in the context of radiosensitivity to propose a potential group that would most likely benefit from the combination of radiotherapy and immunotherapy.

\section{Materials and Methods}

\section{Data sets}

Transcriptome profiling data and clinical data were obtained from the TCGA. We downloaded these data from UCSC Xena (https://xenabrowser.net/ datapages/) using the categories of gene expression RNAseq (IlluminaHiSeq) and phenotype (phenotype). These transcriptome profiling data include gene-level transcription estimates reported as $\log 2(x+1)-$ transformed RSEM normalized counts. We chose head and neck cancer with transcriptome profiling data available for cancer and normal tissue samples. The phenotype sample types were "Primary Tumor" and "solid normal tissue". A data set of normalized results representing gene expression was acquired. A total of 604 samples were collected in the data set, which was accessed during January 2018. These samples were matched with the accompanying data of clinical information. We excluded samples with no survival data $(\mathrm{n}=3)$, no transcriptome profiling data $(n=8)$, and metastatic data $(n=2)$. In total, 517 primary tumor samples and 74 solid normal tissue samples were included in the study. The total population $(\mathrm{n}=517)$ consisted of 289 patients $(55.9 \%)$ who received radiotherapy, 158 patients $(30.6 \%)$ without radiotherapy and 70 patients $(13.5 \%)$ with unknown treatment. We employed CD274 mRNA expression level as a surrogate of PD-L1 and PDCD1 mRNA as a surrogate of PD-1. The HPV status was determined using an HPV in situ hybridization (ISH) test, and the p16 status was assessed using p16 staining. Survival 
data, such as recurrence-free survival (RFS) and overall survival (OS), were obtained from the records in TCGA data set.

\section{The definition of radiosensitivity and clustering analysis}

The radiosensitivity of samples was predicted by a 31-gene signature [10]. The radiosensitivity genes were identified in NCI-60 cancer cells. Based on the correlations between the gene expression levels and the survival fraction at 2Gy (SF2), the 31-gene signature was defined. In summary, 21 genes were upregulated and 10 genes were downregulated in radioresistant (RR) cells relative to radiosensitive (RS) cells (Table S5).

The K-mean clustering [11] was employed to cluster patients into two groups, with $\mathrm{k}=2$, based on the gene expression level of the 31 genes. Clusters were identified as the radioresistant group (RR group) or the radiosensitive group (RS group) based on their prognosis when RT was performed.

\section{Immunohistochemistry}

A total of 114 biopsy samples from patients with primary oropharyngeal cancer were collected at the First Hospital of China Medical University between 2005 and 2011. All the patients accepted radical radiotherapy. Using $4 \mu$ m-thick sections, immunohistochemistry was performed. The primary antibody was PD-L1 (mouse anti-human PD-L1 monoclonal, dilution 1:100, Abcam). The percentage of positive cells and staining intensity were estimated. Consistent with previous reports [12], staining in more than $5 \%$ of the tumor cells was considered positive. For detection of p16, the primary antibody was p16-INK4 (mouse anti-human p16 monoclonal, dilution 1:50, BD Pharmingen ${ }^{\mathrm{TM}}$ ). P16 expression was considered positive if nuclear and cytoplasmic staining in $20 \%$ or more of the tumor cells [13]. The method applied is described in detail in our previous study [14].

\section{Statistical analysis}

Data distributions were tested using the Kolmogorov-Smirnov test. Parametric or nonparametric tests were employed according to the results. Propensity score matching (PSM) was used to balance the bias of RR and RS groups. The chi-square test and the McNemar's test were performed to contrast clinical and pathologic characteristics between RR and RS groups. PD-L1 and PD-1 expression was compared based on radiosensitivity and p16 status using unpaired $t$ test and ANOVA for continues variables. Paired $t$ test and unpaired $t$ test were employed to investigate the gene expression difference between tumor and normal tissue. The rate of survival was analyzed among the three groups based on the following expression levels: 1 ) $<25$ th percentile; 2) 25th to 75th percentile; and 3) $\geq 75$ th percentile. Gehan-Breslow-Wilcoxon tests were used to determine whether the time to event was significantly different between the highest $(\geq 75$ th percentile) and lowest ( $<25$ th percentile) groups [15]. Based on results in univariate analyses, Cox proportional hazards regression models were established. Two-tailed P-values less than 0.05 were considered significant statistically. Statistical analyses were performed using IBM SPSS Statistics software version 22 and GraphPad Prism software 7.00.

\section{Results}

\section{Validation of gene signature related to radiosensitivity}

In previous studies, PD-L1 expression was significantly increased in tumor tissues compared with para-tumor tissues in different cancers $[16,17]$. Similarly, in our present study, PD-L1 expression is upregulated in tumor tissue (unpaired $t$ test, $\mathrm{P}=0.0363$; paired $\mathrm{t}$ test, $\mathrm{P}=0.0584$ ). In addition, no significant difference in PD-1 expression was observed between tumor and para-tumor tissue (Figure S1). Furthermore, PD-L1 expression was significantly positively correlated with PD-1 expression in 517 samples (Pearson Correlation Coefficient, 0.515; $\mathrm{P}<0.0001$, Figure S2).

Recent studies have assessed the role of PD-1/PD-L1 in patients with HNC, but the relationship between PD-1/PD-L1 and radiosensitivity remained unexplored. Herein, we divided all the patients $(n=517)$ into two clusters with different radiosensitivities, cluster $1 \quad(\mathrm{n}=302,58.41 \%)$ and cluster $2(n=215,41.59 \%)$, based on a gene signature related to radiosensitivity (Figure 1). In the context of radiotherapy, patients in the cluster 2 versus cluster 1 exhibited a prolonged overall survival (OS) (HR, $0.6089 ; 95 \% \mathrm{CI}, 0.4045$ to $0.9167 ; \mathrm{P}=0.0026)$ and recurrence-free survival (RFS) (HR, 0.6647; 95\% CI, 0.4155 to $1.063 ; \mathrm{P}=0.0121$ ) over a 200 -month follow-up period. Thus, cluster 1 was identified to be RR group and cluster 2 was RS group. Without radiotherapy, no survival differences were observed between two clusters (Figure 1).

\section{Clinicopathological characteristics based on radiosensitivity}

The clinicopathological features of patients are presented in Table 1. The median follow-up time was 34.75 months (0.07-210.97 months). 


\section{A}

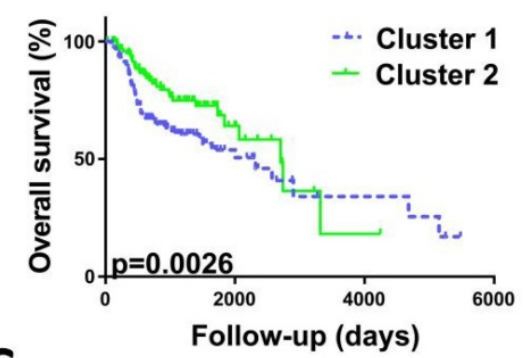

C

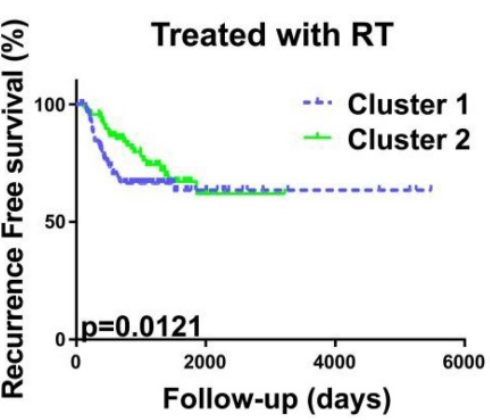

E

Treated with RT

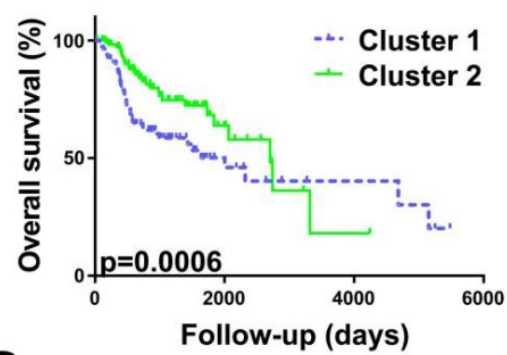

G

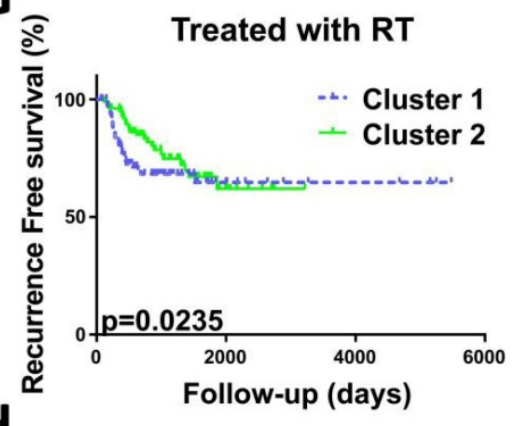

H

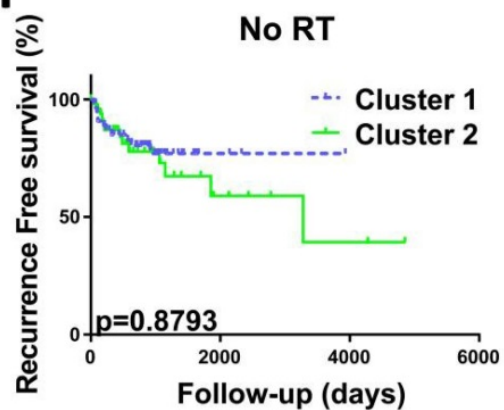

B

No RT

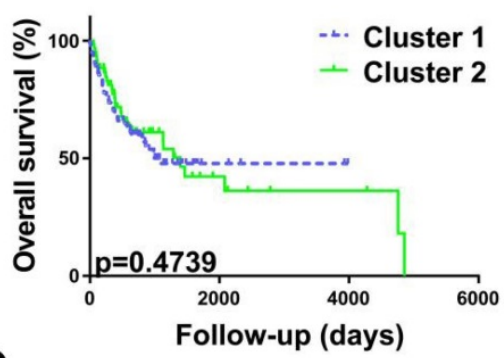

D

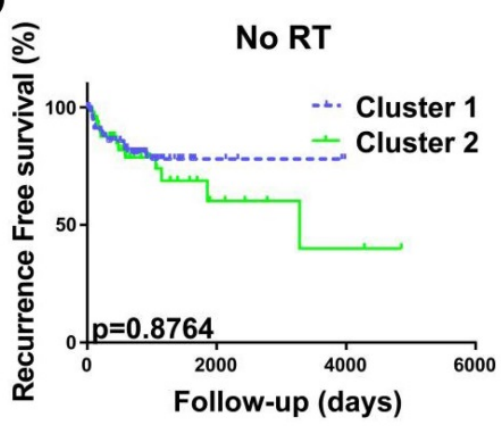

$\mathbf{F}$

No RT

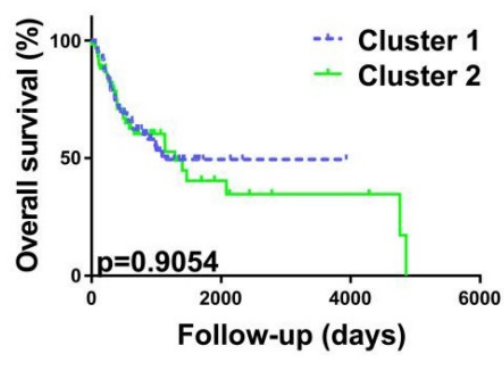

Cluster Cluster $1 \quad$ Cluster 2

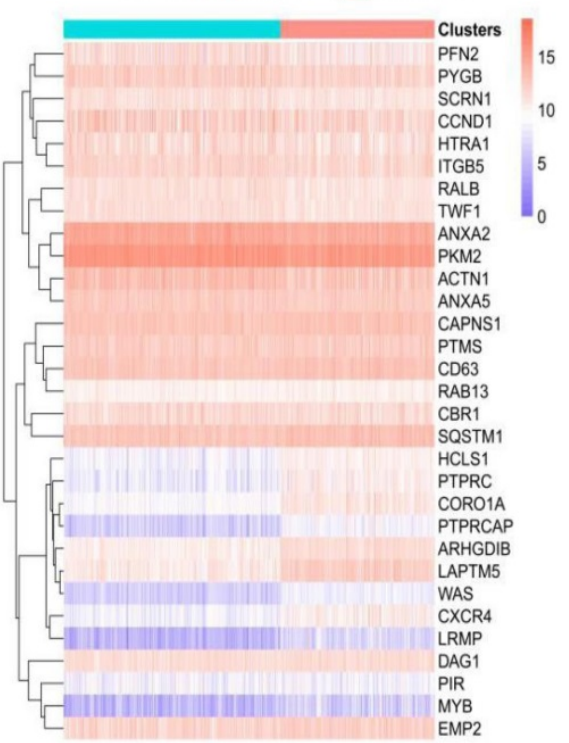

Figure 1. Validation of radiosensitivity gene signature in HNCs. Before PSM analysis, OS curve (A) and RFS curve (C) indicated the prolonged survival of patients with radiotherapy in cluster 2 . $P=0.0026$ and $P=0.0121$, respectively. OS curve $(B)$ and RFS curve (D) indicated no correlation between clusters and survival in patients not treated with radiotherapy. $P=0.4739$ and $P=0.8764$, respectively. After $P S M$ analysis, OS curve $(E)$ and RFS curve $(G)$ indicated the prolonged survival of patients with radiotherapy in cluster 2. $P=0.0006$ and $P=0.0235$, respectively. OS curve $(F)$ and RFS curve $(H)$ indicated no correlation between clusters and survival in patients not treated with radiotherapy. $\mathrm{P}=0.9054$ and $\mathrm{P}=0.8793$, respectively. $\mathrm{P}$-values calculated using the Gehan-Breslow-Wilcoxon test. (I) Heatmap illustrating the relationship between the radiosensitivity gene signature of 31 genes and two groups before PSM analysis. Columns represent samples and radiosensitivity, and rows indicate genes expressed. Legend: blue, low expression; pink, high expression; green, cluster 1 group; pink, cluster 2 group. 
Table 1. Clinicopathological features according to radiosensitivity before PSM.

\begin{tabular}{|c|c|c|c|c|c|c|c|c|}
\hline & & \multicolumn{2}{|c|}{ RR(Cluster1) } & \multicolumn{2}{|c|}{ RS(Cluster2) } & \multicolumn{2}{|c|}{ Total } & \multirow[t]{2}{*}{ P-value } \\
\hline & & $\mathrm{N}$ & $\%$ & $\mathrm{~N}$ & $\%$ & $\mathrm{~N}$ & $\%$ & \\
\hline \multirow[t]{2}{*}{ Age } & $<60$ & 139 & 46.0 & 92 & 42.8 & 231 & 44.7 & $0.466^{a}$ \\
\hline & $\geq 60$ & 163 & 54.0 & 123 & 57.2 & 286 & 55.3 & \\
\hline \multirow[t]{2}{*}{ Gender } & Female & 70 & 23.2 & 65 & 30.2 & 135 & 26.1 & 0.072 \\
\hline & Male & 232 & 76.8 & 150 & 69.8 & 382 & 73.9 & \\
\hline \multirow[t]{3}{*}{ Alcohol } & No & 92 & 30.5 & 70 & 66.5 & 162 & 31.3 & 0.265 \\
\hline & Yes & 201 & 66.6 & 143 & 32.6 & 344 & 66.5 & \\
\hline & unknown & 9 & 3 & 2 & 0.9 & 11 & 2.1 & \\
\hline \multirow[t]{2}{*}{ Packs/year } & $<40$ & 203 & 67.2 & 148 & 68.8 & 351 & 67.9 & 0.698 \\
\hline & $\geq 40$ & 99 & 32.8 & 67 & 31.2 & 166 & 32.1 & \\
\hline \multirow[t]{3}{*}{ HPV } & Negative & 35 & 11.6 & 30 & 14 & 65 & 12.6 & $<0.0001$ \\
\hline & Positive & 3 & 1 & 18 & 8.4 & 21 & 4.1 & \\
\hline & unknown & 264 & 87.4 & 167 & 77.7 & 431 & 83.4 & \\
\hline \multirow[t]{3}{*}{ P16 } & Negative & 39 & 12.9 & 33 & 15.3 & 72 & 13.9 & $<0.0001$ \\
\hline & Positive & 9 & 3 & 29 & 13.5 & 38 & 7.4 & \\
\hline & unknown & 254 & 84.1 & 153 & 71.2 & 407 & 78.7 & \\
\hline \multirow[t]{3}{*}{ EGFR status } & Unamplified & 4 & 1.3 & 12 & 5.6 & 16 & 3.1 & 0.006 \\
\hline & Amplified & 9 & 3 & 2 & 0.9 & 11 & 2.1 & \\
\hline & unknown & 289 & 95.7 & 201 & 93.5 & 490 & 94.8 & 0.595 \\
\hline \multirow[t]{3}{*}{ Anatomic } & Hypopharynx & 6 & 2 & 4 & 1.9 & 10 & 1.9 & \\
\hline & Larynx & 63 & 20.9 & 53 & 24.7 & 116 & 22.4 & \\
\hline & Oral & 233 & 77.2 & 158 & 73.5 & 391 & 75.6 & \\
\hline \multirow[t]{6}{*}{ Grade } & G1 & 43 & 14.2 & 19 & 8.8 & 62 & 12.0 & $<0.0001$ \\
\hline & G2 & 192 & 63.6 & 109 & 50.7 & 301 & 58.2 & \\
\hline & G3 & 58 & 19.2 & 67 & 31.2 & 125 & 24.2 & \\
\hline & G4 & 1 & 0.3 & 6 & 2.8 & 7 & 1.4 & \\
\hline & GX & 6 & 2 & 12 & 5.6 & 18 & 3.5 & \\
\hline & unknown & 2 & 0.7 & 2 & 0.9 & 4 & 0.8 & \\
\hline \multirow[t]{5}{*}{ Treatment } & Surgery & 60 & 19.9 & 40 & 18.6 & 100 & 19.3 & 0.723 \\
\hline & Radiation & 24 & 7.9 & 12 & 5.6 & 36 & 7 & \\
\hline & Concurrent & 22 & 7.3 & 21 & 9.8 & 43 & 8.3 & \\
\hline & Non-concurrent & 4 & 1.3 & 3 & 1.4 & 7 & 1.4 & \\
\hline & unknown & 192 & 63.6 & 139 & 64.7 & 331 & 64 & \\
\hline \multirow[t]{3}{*}{ Radiation } & Yes & 164 & 54.3 & 125 & 58.1 & 289 & 55.9 & 0.684 \\
\hline & No & 96 & 31.8 & 62 & 28.8 & 158 & 30.6 & \\
\hline & unknown & 42 & 13.9 & 28 & 13 & 70 & 13.5 & \\
\hline \multirow[t]{3}{*}{ Margin } & Negative/Close & 245 & 81.1 & 152 & 70.7 & 397 & 76.8 & 0.002 \\
\hline & Positive & 34 & 11.3 & 25 & 11.6 & 59 & 11.4 & \\
\hline & unknown & 23 & 7.6 & 38 & 17.7 & 61 & 11.8 & \\
\hline \multirow[t]{3}{*}{ CD274 } & High & 48 & 15.9 & 81 & 37.7 & 129 & 25 & $<0.0001$ \\
\hline & Low & 98 & 32.5 & 31 & 14.4 & 129 & 25 & \\
\hline & Medium & 156 & 51.7 & 103 & 47.9 & 259 & 50.1 & \\
\hline \multirow[t]{3}{*}{ PDCD1 } & High & 13 & 4.3 & 116 & 54 & 129 & 25 & $<0.0001$ \\
\hline & Low & 124 & 41.1 & 5 & 2.3 & 129 & 25 & \\
\hline & medium & 165 & 54.6 & 94 & 43.7 & 259 & 50.1 & \\
\hline
\end{tabular}

a. Chi-square test (and the Fisher's exact test when appropriate)

Abbreviations: Alcohol, alcohol history; Packs/year, packs/year smoked, HPV, HPV status assessed by ISH testing; P16, HPV status assessed by p16 testing; EGFR status, Epidermal growth factor receptor amplification status; Anatomic, anatomic neoplasm subdivision; Grade, neoplasm histologic grade; Concurrent, concurrent chemotherapy;

Non-concurrent, chemotherapy not administered concurrently; RR, radioresistant group; RS, radiosensitive group.

Between the RR and RS groups, the significantly different factors include HPV status $(\mathrm{P}<0.0001)$, p16 status $(\mathrm{P}<0.0001)$, EGFR amplification status $(\mathrm{P}=0.006)$, pathologic $\mathrm{T}(\mathrm{P}<0.0001)$, pathologic $\mathrm{N}$ $(\mathrm{P}<0.0001)$, neoplasm histologic grade $(\mathrm{P}<0.0001)$, lymphovascular invasion present $(\mathrm{P}=0.013)$, targeted molecular therapy $(\mathrm{P}=0.021)$, margin status $(\mathrm{P}=0.002)$, PD-L1 expression level $(\mathrm{P}<0.0001)$, and PD-1 expression level $(\mathrm{P}<0.0001)$. Similar to previous studies $[7,18]$, we found that patients positive for $\mathrm{HPV} / \mathrm{p} 16$ were more abundant in the RS group compared with the RR group $(\mathrm{P}<0.0001)$, whereas patients with EGFR amplification were more common in the RR group compared with the RS group $(\mathrm{P}=0.006)$. After PSM analysis, two groups had similar clinicopathologic features (Table 2). And survival analysis still showed different OS $(\mathrm{P}=0.0006)$ and RFS ( $p=0.0235)$ between RR group and the RS group (Figure 1).

\section{Factors correlated with PD-LI and PD-1 expression: radiosensitivity and HPV/p16 status}

Fiedler $\mathrm{M}$ et al recently reported that high PD-L1 expression promoted increased radiotherapy response rates without reaching statistical significance [7]. Similarly, significant differences in PD-L1 and PD-1 status were noted between the RR and RS groups (Table 2). Given the relationship between PD-L1 and PD-1 expression and radiosensitivity, we next assessed factors associated with PD-L1 and PD-1 expression via variance analysis (Table 3). In 
univariate analysis, pathologic stage $(\mathrm{P}=0.019)$ and radiosensitivity $(\mathrm{P}<0.0001)$ were significant factors associated with PD-L1 expression, whereas p16 $(\mathrm{P}=0.0002)$, neoplasm histologic grade $(\mathrm{P}=0.001)$ and radiosensitivity $(\mathrm{P}<0.0001)$ were significantly associated with PD-1 expression. In multivariate analysis, pathologic stage $(\mathrm{P}=0.0128)$ was a significant factor associated with PD-L1 expression, and p16 status $(\mathrm{P}=0.0332)$ was a significant factor associated with PD-1 expression. Before and after PSM analysis, PD-L1 and PD-1 expression levels are significantly increased in the RS group compared with the RR group $(\mathrm{P}<0.0001)$ (Figure 2$)$.

Table 2. Clinicopathological features according to radiosensitivity after PSM

\begin{tabular}{|c|c|c|c|c|c|c|c|c|}
\hline & & \multicolumn{2}{|c|}{ RR(Cluster1) } & \multicolumn{2}{|c|}{ RS(Cluster2) } & \multicolumn{2}{|c|}{ Total } & \multirow[t]{2}{*}{ P-value } \\
\hline & & $\mathrm{N}$ & $\%$ & $\mathrm{~N}$ & $\%$ & $\mathrm{~N}$ & $\%$ & \\
\hline \multirow[t]{2}{*}{ Age } & $<60$ & 89 & 45.9 & 80 & 41.2 & 169 & 43.6 & $0.431^{\mathrm{a}}$ \\
\hline & $\geq 60$ & 105 & 54.1 & 114 & 58.8 & 219 & 56.4 & \\
\hline \multirow[t]{2}{*}{ Gender } & Female & 47 & 24.2 & 60 & 30.9 & 107 & 27.6 & 0.154 \\
\hline & Male & 147 & 75.8 & 134 & 69.1 & 281 & 72.4 & \\
\hline \multirow[t]{3}{*}{ Alcohol } & No & 59 & 30.4 & 62 & 32 & 121 & 31.2 & 0.570 \\
\hline & Yes & 129 & 66.5 & 130 & 67 & 259 & 66.8 & \\
\hline & unknown & 6 & 3.1 & 2 & 1 & 8 & 2.1 & \\
\hline \multirow[t]{2}{*}{ Packs/year } & $<40$ & 131 & 67.5 & 130 & 67 & 261 & 67.3 & 1.000 \\
\hline & $\geq 40$ & 63 & 32.5 & 64 & 33 & 127 & 32.7 & \\
\hline \multirow[t]{3}{*}{$\mathrm{HPV}$} & Negative & 24 & 12.4 & 28 & 14.4 & 52 & 13.4 & 0.075 \\
\hline & Positive & 3 & 1.5 & 12 & 6.2 & 15 & 3.9 & \\
\hline & unknown & 167 & 86.1 & 154 & 79.4 & 321 & 82.7 & \\
\hline \multirow[t]{3}{*}{ P16 } & Negative & 27 & 13.9 & 31 & 16 & 58 & 14.9 & 0.018 \\
\hline & Positive & 6 & 3.1 & 19 & 9.8 & 25 & 6.4 & \\
\hline & unknown & 161 & 83 & 144 & 74.2 & 305 & 78.6 & \\
\hline \multirow[t]{3}{*}{ EGFR status } & Unamplified & 4 & 1.3 & 11 & 5.7 & 15 & 3.9 & 0.067 \\
\hline & Amplified & 7 & 3.6 & 1 & 0.5 & 8 & 2.1 & \\
\hline & unknown & 183 & 94.3 & 182 & 93.8 & 365 & 94.1 & \\
\hline \multirow[t]{3}{*}{ Anatomic } & Hypopharynx & 3 & 1.5 & 4 & 2.1 & 7 & 1.8 & 0.670 \\
\hline & Larynx & 39 & 20.1 & 49 & 25.3 & 88 & 22.7 & \\
\hline & Oral & 152 & 78.4 & 141 & 72.7 & 293 & 75.5 & \\
\hline \multirow[t]{6}{*}{ Grade } & G1 & 19 & 9.8 & 19 & 9.8 & 38 & 9.8 & 0.780 \\
\hline & G2 & 112 & 57.7 & 105 & 54.1 & 217 & 55.9 & \\
\hline & G3 & 54 & 27.8 & 60 & 30.9 & 114 & 29.4 & \\
\hline & G4 & 1 & 0.5 & 2 & 1 & 3 & 0.8 & \\
\hline & GX & 6 & 3.1 & 7 & 3.6 & 13 & 3.4 & \\
\hline & unknown & 2 & 1 & 1 & 0.5 & 3 & 0.8 & \\
\hline \multirow[t]{5}{*}{ Treatment } & Surgery & 41 & 21.1 & 38 & 19.6 & 79 & 20.4 & 0.596 \\
\hline & Radiation & 14 & 7.2 & 12 & 6.2 & 26 & 6.7 & \\
\hline & Concurrent & 20 & 10.3 & 15 & 7.7 & 35 & 9 & \\
\hline & Non-concurrent & 2 & 1 & 1 & 0.5 & 3 & 0.8 & \\
\hline & unknown & 117 & 60.3 & 128 & 66 & 245 & 63.1 & \\
\hline Radiation & Yes & 111 & 57.2 & 111 & 57.2 & 222 & 57.2 & 0.127 \\
\hline & No & 62 & 32 & 60 & 30.9 & 122 & 31.4 & \\
\hline & unknown & 21 & 10.8 & 23 & 11.9 & 44 & 11.3 & \\
\hline Margin & Negative/Close & 144 & 74.2 & 144 & 74.2 & 288 & 74.2 & 0.947 \\
\hline & Positive & 28 & 14.4 & 25 & 12.9 & 53 & 13.7 & \\
\hline & unknown & 22 & 11.3 & 38 & 12.9 & 47 & 12.1 & \\
\hline T-stage & $\mathrm{T} 1$ & 15 & 7.7 & 22 & 11.3 & 37 & 9.5 & 0.087 \\
\hline & $\mathrm{T} 2$ & 51 & 26.3 & 55 & 28.4 & 106 & 27.3 & \\
\hline & T3 & 55 & 28.4 & 30 & 15.5 & 85 & 21.9 & \\
\hline & $\mathrm{T} 4$ & 51 & 26.3 & 59 & 30.4 & 110 & 28.4 & \\
\hline & $\mathrm{TX}$ & 16 & 8.2 & 18 & 9.3 & 34 & 8.8 & \\
\hline & unknown & 6 & 3.1 & 10 & 5.2 & 16 & 4.1 & \\
\hline $\mathrm{N}$-stage & No & 66 & 34 & 53 & 27.3 & 119 & 30.7 & 0.388 \\
\hline & N1 & 24 & 12.4 & 29 & 14.9 & 53 & 13.7 & \\
\hline & N2 & 62 & 32 & 62 & 32 & 124 & 32 & \\
\hline & N3 & 3 & 1.5 & 4 & 2.1 & 7 & 1.8 & \\
\hline & $\mathrm{NX}$ & 32 & 16.5 & 35 & 18 & 67 & 17.3 & \\
\hline & unknown & 7 & 3.6 & 11 & 5.7 & 18 & 4.6 & \\
\hline Invasion & No & 83 & 42.8 & 78 & 40.2 & 161 & 41.5 & 0.323 \\
\hline & Yes & 55 & 28.4 & 45 & 23.2 & 100 & 25.8 & \\
\hline & unknown & 56 & 28.9 & 71 & 36.6 & 127 & 32.7 & \\
\hline Targeted & No & 100 & 51.5 & 92 & 47.4 & 192 & 49.5 & 0.732 \\
\hline & Yes & 59 & 30.4 & 63 & 32.5 & 122 & 31.4 & \\
\hline & unknown & 35 & 18 & 39 & 20.1 & 74 & 19.1 & \\
\hline CD274 & High & 32 & 16.5 & 65 & 33.5 & 97 & 25 & $<0.0001$ \\
\hline & Low & 63 & 32.5 & 34 & 17.5 & 97 & 25 & \\
\hline & Medium & 99 & 51 & 95 & 49 & 194 & 50 & \\
\hline
\end{tabular}




\begin{tabular}{|c|c|c|c|c|c|c|c|c|}
\hline & & \multicolumn{2}{|c|}{ RR(Cluster1) } & \multicolumn{2}{|c|}{ RS(Cluster2) } & \multicolumn{2}{|c|}{ Total } & \multirow[t]{2}{*}{ P-value } \\
\hline & & $\mathrm{N}$ & $\%$ & $\mathrm{~N}$ & $\%$ & $\mathrm{~N}$ & $\%$ & \\
\hline \multirow[t]{3}{*}{ PDCD1 } & High & 8 & 4.1 & 89 & 45.9 & 97 & 25 & $<0.0001$ \\
\hline & Low & 89 & 45.9 & 8 & 4.1 & 97 & 25 & \\
\hline & medium & 97 & 50 & 94 & 50 & 194 & 50 & \\
\hline
\end{tabular}

a. McNemar's Test (and the Bowker's Test when appropriate)

Abbreviations: Alcohol, alcohol history; Packs/year, packs/year smoked, HPV, HPV status assessed by ISH testing; P16, HPV status assessed by p16 testing; EGFR status, Epidermal growth factor receptor amplification status; Anatomic, anatomic neoplasm subdivision; Grade, neoplasm histologic grade; Concurrent, concurrent chemotherapy; Non-concurrent, chemotherapy not administered concurrently; Invasion, lymphovascular invasion present; Targeted, targeted molecular therapy; RR, radioresistant group; RS, radiosensitive group.

Table 3. Univariate and multivariate variance analysis for PD-L1 and PD-1 expression.

\begin{tabular}{|c|c|c|c|c|c|c|c|c|c|}
\hline & & PD-L1 & & & & PD-1 & & & \\
\hline & & Univariate & & Multivariate & & Univariate & & Multivariate & \\
\hline Variables & & Mean (SD) & p-value & $\mathrm{F}$ & p-value & Mean (SD) & p-value & $\mathrm{F}$ & p-value \\
\hline \multirow[t]{2}{*}{ Age } & $<60$ & $6.20(1.55)$ & 0.054 & & & $5.24(1.76)$ & 0.164 & & \\
\hline & $>=60$ & $6.48(1.69)$ & & & & $5.45(1.55)$ & & & \\
\hline \multirow[t]{2}{*}{ P16 } & Negative & $6.55(1.54)$ & 0.860 & & & $5.30(1.64)$ & 0.0002 & 4.66 & 0.033 \\
\hline & Positive & $6.45(1.66)$ & & & & $6.62(1.85)$ & & & \\
\hline \multirow[t]{4}{*}{ Stage } & I & $7.17(1.68)$ & 0.019 & 3.64 & 0.013 & $5.70(1.34)$ & 0.407 & & \\
\hline & II & $6.67(1.55)$ & & & & $5.42(1.83)$ & & & \\
\hline & III & $6.34(1.82)$ & & & & $5.29(1.61)$ & & & \\
\hline & IV & $6.21(1.54)$ & & & & $5.20(1.62)$ & & & \\
\hline \multirow[t]{2}{*}{ EGFR } & Unamplified & $6.50(1.50)$ & 0.680 & & & $5.74(1.70)$ & 0.211 & & \\
\hline & Amplified & $6.95(1.92)$ & & & & $4.85(1.87)$ & & & \\
\hline \multirow[t]{5}{*}{ Grade } & GX & $6.69(1,23)$ & 0.723 & & & $6.07(2.15)$ & 0.001 & & \\
\hline & G1 & $6.53(1.67)$ & & & & $5.27(1.58)$ & & & \\
\hline & G2 & $6.28(1.58)$ & & & & $5.14(1.55)$ & & & \\
\hline & G3 & $6.40(1.75)$ & & & & $5.74(1.70)$ & & & \\
\hline & G4 & $6.41(1.30)$ & & & & $6.53(1.57)$ & & & \\
\hline \multirow[t]{2}{*}{ Radiosensitivity } & RR & $4.44(1.30)$ & $<0.0001$ & 32.35 & $<0.0001$ & $6.01(1.58)$ & 0.0001 & 96.04 & $<0.0001$ \\
\hline & RS & $6.64(1.15)$ & & & & $6.85(1.62)$ & & & \\
\hline
\end{tabular}

Abbreviations: Grade, neoplasm histologic grade; EGFR, Epidermal growth factor receptor amplification status; RR, radioresistant group; RS, radiosensitive group.

Given that HPV/p16 status is associated with radiosensitivity and directly associated with cancer immunogenicity. We then analyzed the impact of HPV and p16 on PD-L1 and PD-1 expression. Before and after PSM analysis, PD-1 expression is significantly increased in the HPV-positive group and p16-positive group (Figure 2). However, we failed to identify a significant relationship between PD-L1 expression and $\mathrm{HPV} / \mathrm{p} 16$ status. For a more comprehensive study, we estimated the correlations between PD-L1 expression and p16 status using immunohistochemistry. Patient biopsy samples $(n=114)$ were assessed for PD-L1 and p16 expression in tumor tissues (Figure 3). PD-L1 positive expression was identified in 37 cases $(32.5 \%)$, and p16 positive expression was identified in 28 cases (24.6\%). Positive p16 status was associated with positive PD-L1 expression $(\mathrm{P}=0.0224)$.

\section{Survival analysis based on radiosensitivity and PD-LI status}

In previous studies, PD-L1 was proven to be a prognostic marker in different cohorts, such as male, smokers and patients with P16 negative status [19, 20]. Thus, in our study, we estimated whether PD-1 and PD-L1 are prognostic markers in patients with different radiosensitivities. We aim to determine the characteristics of patients who could benefit from
anti-PD-1/PD-L1 therapy combined with radiotherapy.

Survival analysis was employed for patients treated with radiotherapy $(n=289)$ stratified by $R S$ and RR groups. As shown in previous studies [21, 22], we found that a negative or close margin status, low pathologic stage and $\mathrm{HPV} / \mathrm{p} 16$ positive status predicted for significantly superior outcome (Table 4). In univariate analysis, PD-L1 high expression was associated with worse OS (HR, 2.196; 95\% CI, 1.081-4.46; $\mathrm{p}=0.0108)$ compared with PD-L1 low expression in the RR group. Interestingly, PD-L1 high expression predicted worse OS in the patients with both $\mathrm{HPV} / \mathrm{p} 16$ negative status and radioresistant gene signature (HPV: HR, 2.334; 95\% CI, 0.7828-6.961; $\mathrm{P}=0.0313$; p16: HR, 2.486; 95\% CI, 0.8559-7.219; $\mathrm{P}=0.0192$ ) (Figure 4). No prognostic impact of PD-L1 expression was noted in the RS group. In contrast, high PD-1 expression was a significant factor in the overall radiotherapy group $(\mathrm{n}=289)$ and predicted better RFS (HR, 0.4892; 95\% CI, 0.2357-1.015; $\mathrm{P}=0.023$ ) (Figure 4). We employed a Cox hazard regression model that included factors that were significant in univariate analysis (Table 5). Margin status, pathologic stage, PD-L1 expression and radiosensitivity status were confirmed as independent prognostic parameters for OS and RFS in different groups. 
A

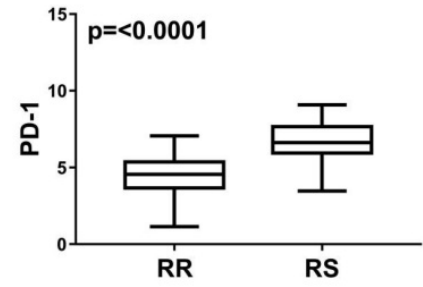

C

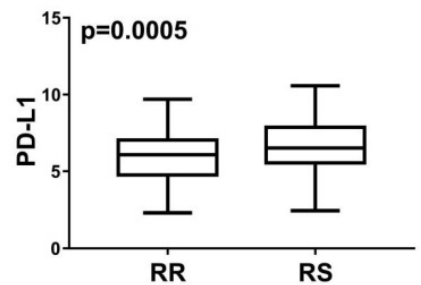

E

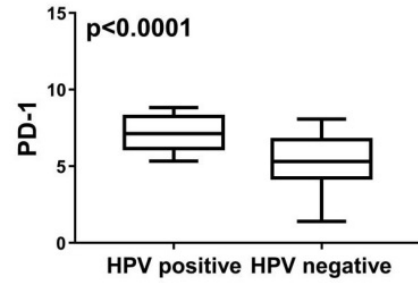

G

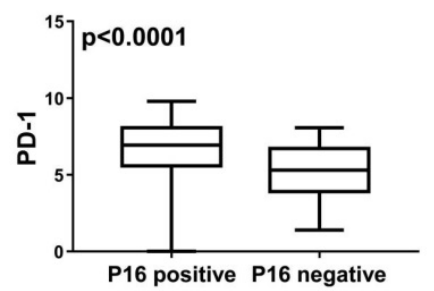

B

After PSM

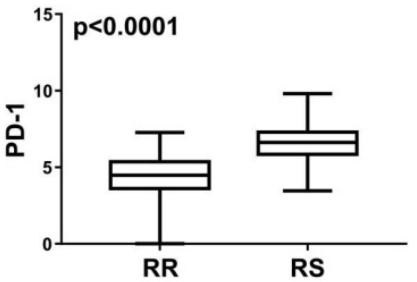

D

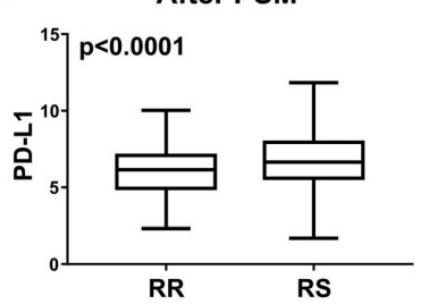

$\mathbf{F}$

After PSM

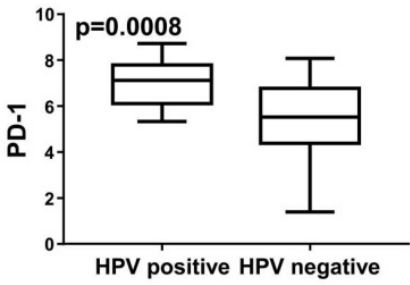

H

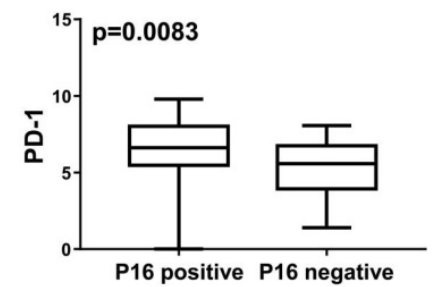

Figure 2. Levels of PD-1 mRNA expression and PD-L1 mRNA expression in head and neck tumors before and after PSM analysis. PD-1 mRNA is upregulated in RS tumors (A), HPV-positive tumors (E) and P16-positive tumors (G) before PSM analysis. All P<0.0001. After PSM analysis, PD-1 mRNA expression is also upregulated in RS tumors (B), HPV-positive tumors $(F)$ and $P 16$-positive tumors $(H)$. $P<0.0001, P=0.0008$ and $P=0.0083$, respectively. PD-L1 mRNA expression is upregulated in RS tumors before (C) and after (D) PSM analysis. $\mathrm{P}=0.0005$ and $\mathrm{P}<0.0001$, respectively.

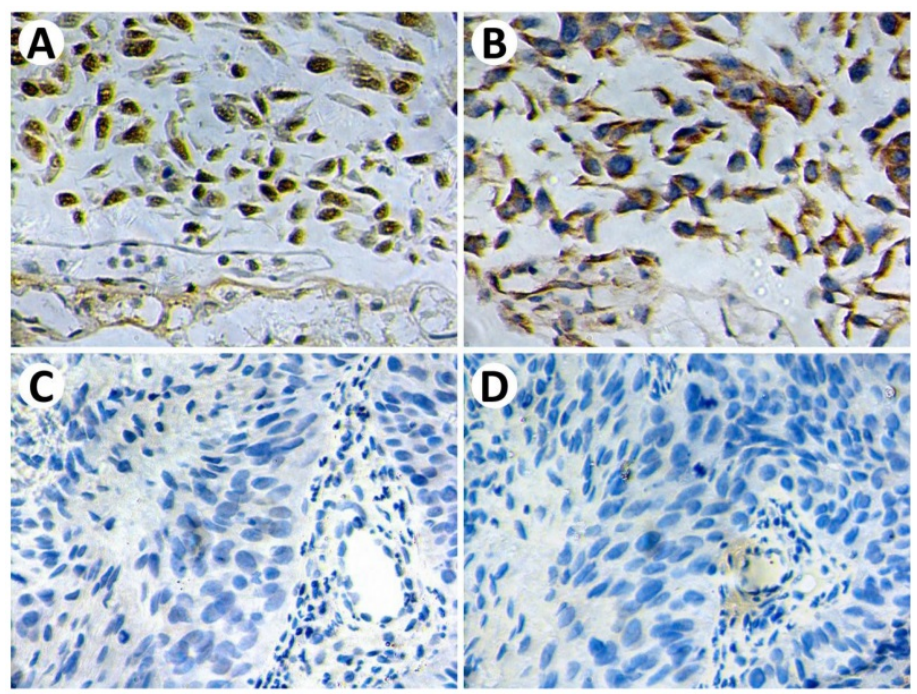

Figure 3. Representative immunohistochemical staining of p16 and PD-LI. PI6-positive staining (A) demonstrates strong PD-L1 expression (B) on tumor cells. P16-negative staining $(C)$ demonstrates negative PD-L1 expression (D) on tumor cells. Original magnification: $\times 400$ 
A

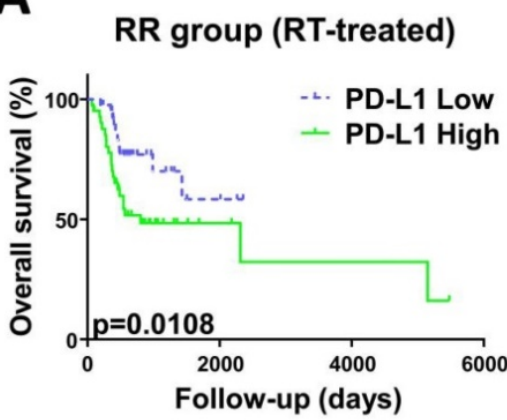

C

HPV-negative (RR group)

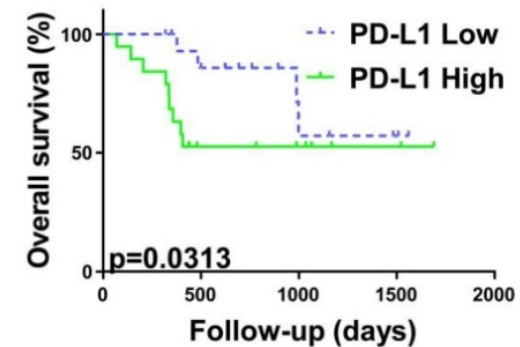

E

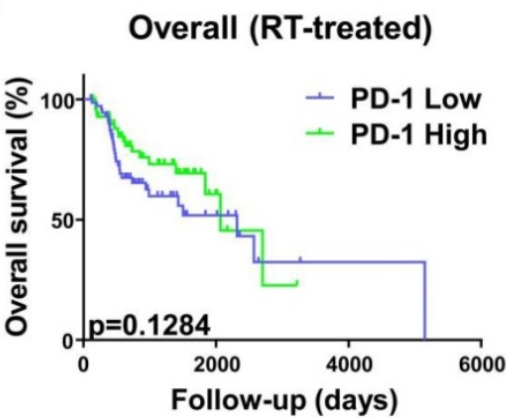

B

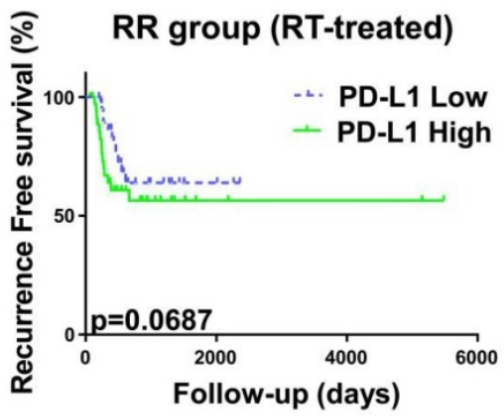

D

\section{P16-negative (RR group)}

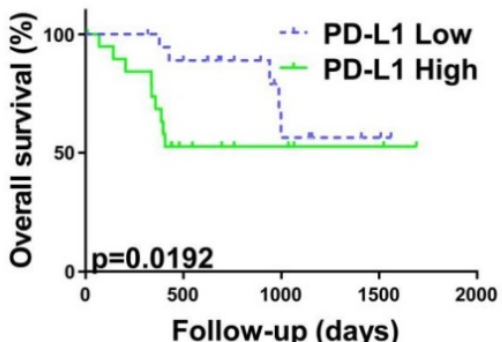

$\mathbf{F}$

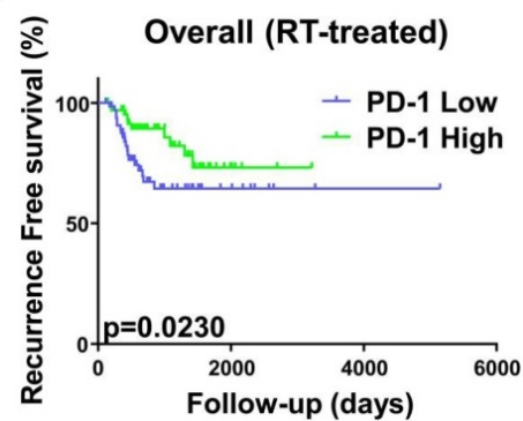

Figure 4. Kaplan-Meier curves analysis stratified by radiosensitivity. OS curve (A) and RFS curve (B) indicated worse prognosis of patients with high PD-L1 mRNA expression in the RR group compared with low PD-L1 mRNA expression. $P=0.0108$ and 0.0687 , respectively. OS curves indicated worse prognosis of HPV (C) or $P 16$ (D) negative status patients with high PD-L1 mRNA expression in the RR group compared with low PD-L1 mRNA expression. $P=0.0313$ and 0.0192 , respectively. (E) OS curves indicated that the PD-1 mRNA level does not correlate with overall survival in all patients $(n=289)$. $P=0.1284$. (F) RFS curves indicated that patients with low $P D-1$ mRNA levels recurred earlier than those with high PD-1 mRNA levels. $\mathrm{P}=0.0230$. $\mathrm{P}$ values calculated by Gehan-Breslow-Wilcoxon test.

Table 4. Univariate analysis for overall survival (OS) and recurrence-free survival (RFS)

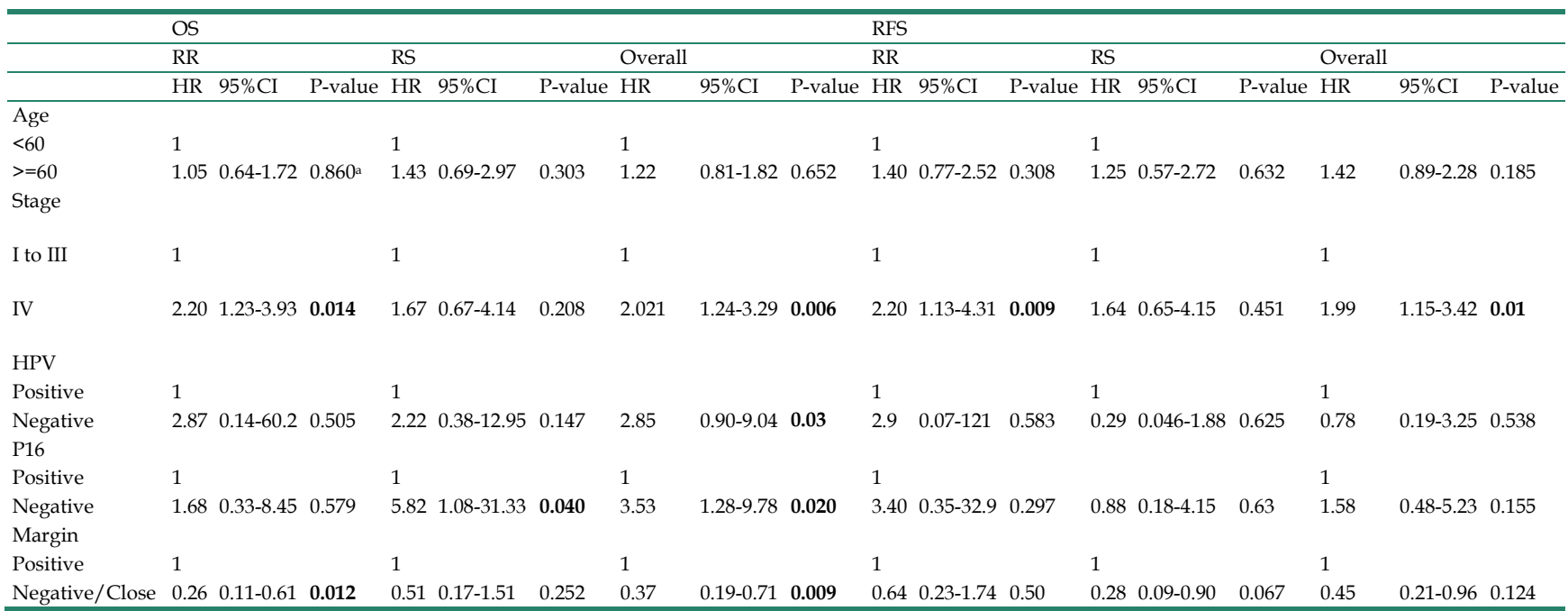




\begin{tabular}{|c|c|c|c|c|c|c|c|c|c|c|c|c|c|c|c|c|c|c|}
\hline & OS & & & & & & & & & RFS & & & & & & & & \\
\hline & RR & & & RS & & & Overall & & & RR & & & RS & & & Over & & \\
\hline & HR & $95 \% \mathrm{CI}$ & P-value & HR & $95 \% \mathrm{CI}$ & $\mathrm{P}$-value & HR & $95 \% \mathrm{CI}$ & $\mathrm{P}$-value & HR & $95 \% \mathrm{CI}$ & $\mathrm{P}$-value & HR & $95 \% \mathrm{CI}$ & $\mathrm{P}$-value & HR & $95 \% \mathrm{CI}$ & P-value \\
\hline \multicolumn{19}{|l|}{ PD-L1 } \\
\hline Low & 1 & & & 1 & & & 1 & & & 1 & & & 1 & & & 1 & & \\
\hline High & 2.20 & $1.08-4.46$ & 0.011 & 1.07 & $0.40-2.88$ & 0.929 & 1.51 & $0.82-2.76$ & 0.134 & 1.67 & $0.76-3.69$ & 0.069 & 0.59 & $0.19-1.82$ & 0.361 & 0.78 & $0.39-1.56$ & 0.650 \\
\hline \multicolumn{19}{|l|}{ PD-1 } \\
\hline Low & 1 & & & 1 & & & 1 & & & 1 & & & 1 & & & 1 & & \\
\hline High & 1.05 & $0.49-2.24$ & 0.440 & 1.29 & $0.47-3.58$ & 0.990 & 0.68 & $0.38-1.21$ & 0.128 & 1.12 & $0.49-2.60$ & 0.417 & 0.37 & $0.10-1.37$ & 0.248 & 0.9 & $0.24-1.02$ & 0.023 \\
\hline \multicolumn{19}{|c|}{ Radiosensitivity } \\
\hline RR & & & & & & & 1 & & & & & & & & & 1 & & \\
\hline RS & & & & & & & 0.61 & $0.40-0.92$ & 0.003 & & & & & & & 0.66 & $0.42-1.06$ & 0.012 \\
\hline
\end{tabular}

a. Gehan-Breslow-Wilcoxon tests were used to determine whether the time to event significantly differed between the high expression group ( $\geq 75 \%$ ) and low expression group $(<25 \%)$ of PD-L1 and PD-1

Abbreviations: HPV, HPV status assessed by ISH testing; P16, HPV status assessed by p16 testing; RR, radioresistant group; RS, radiosensitive group.

Table 5. Cox hazard regression models for overall survival (OS) and recurrence-free survival (RFS)

\begin{tabular}{|c|c|c|c|c|c|c|c|}
\hline & & OS & & & RFS & & \\
\hline & & HR & $95 \% \mathrm{CI}$ & P-value & HR & $95 \% \mathrm{CI}$ & P-value \\
\hline \multicolumn{8}{|l|}{ RR } \\
\hline Stage & I to IV (Incremental) & 2.22 & $1.30-3.80$ & 0.004 & 2.13 & $1.15-3.93$ & 0.016 \\
\hline Margin & Positive (vs. Negative/close) & 2.72 & $1.42-5.21$ & 0.003 & & & \\
\hline PD-L1 & High (vs. Low) & 1.33 & $1.07-1.66$ & 0.010 & 1.23 & $0.98-1.54$ & 0.077 \\
\hline \multicolumn{8}{|l|}{ RS } \\
\hline P16 & Positive (vs. Negative) & 0.02 & $0.00-18.98$ & 0.261 & & & \\
\hline \multicolumn{8}{|l|}{ Overall } \\
\hline Stage & I to IV (Incremental) & 1.80 & $1.19-2.72$ & 0.005 & 1.63 & $1.05-2.51$ & 0.029 \\
\hline Margin & Positive (vs. Negative/close) & 2.27 & $1.33-3.87$ & 0.003 & & & \\
\hline PD-1 & High (vs. Low) & & & & 0.97 & $0.78-1.21$ & 0.795 \\
\hline Radiosensitivity & RR (vs. RS) & 1.76 & $1.09-2.85$ & 0.021 & 1.43 & $0.70-2.91$ & 0.330 \\
\hline
\end{tabular}

Abbreviations: HPV, HPV status assessed by ISH testing; P16, HPV status assessed by p16 testing; RR, radioresistant group; RS, radiosensitive group.

For a more comprehensive study, we also evaluated the prognostic impact of radiosensitivity stratified by PD-L1 (Table S1) and PD-1 expression level (Table S2). In univariate analysis, in the PD-L1 high group, the RS group exhibited prolonged overall survival time (HR, 0.4858; 95\% CI, 0.2136-1.105; $\mathrm{P}=0.0189)$ and reduced risk of recurrence $(\mathrm{HR}, 0.4371$; 95\% CI, 0.1421-1.345; $\mathrm{P}=0.0231$ ) (Figure S3). No effect on OS and RFS was noted in the PD-L1 low group and overall group. This survival difference in the PD-L1 high group further validated the negative prognostic value of PD-L1 in the RR group and suggested the potential to reverse radioresistance through anti-PD-L1 therapy. Significant factors in univariate analysis and radiosensitivity status were taken into account in the multivariable analysis (Table S3).

\section{Discussion}

PD-1 is expressed on activated B cells, CD4 and CD8 T cells, dendritic cells, natural killer cells, APCs and monocytes. In contrast, PD-L1 is widely expressed on B cells, $\mathrm{T}$ cells, dendritic cells, mesenchymal stem cells, macrophages and a wide variety of non-hematopoietic cells. Of note, PD-L1 is also expressed on various tumor cells, causing a negative effect on antitumor immunity [23].

Radiation-induced DNA damage causes cell death. In addition to the direct action on tumor cells, radiation also plays a role in immunomodulation. Radiation produces immunogenicity via dendritic cells, which consume tumor peptides of dead tumor cells and recruit cytotoxic T lymphocytes (CTL) into the tumor microenvironment. To escape the immunologic responses of the host, PD-L1 was expressed by tumor cells and induced T cell apoptosis [24]. Thus, the predictive value of basal PD-1/PD-L1 expression is our target.

As shown in previous studies [24, 25], the gene signature related to radiosensitivity [10] was associated with OS in glioma and RFS in breast cancer. Of note, in our work, the RS group exhibited both better OS and RFS rates compared with the RR group when patients received radiotherapy. The differences were not significant for patients who were not treated with radiotherapy. Therefore, the gene signature is a predictive marker for head and neck cancer patients who received radiotherapy.

To define the factors that impact radiosensitivity, we analyzed patient and tumor characteristics in RS and RR groups. In our study, alleged favorable prognostic factors for HNC, such as HPV-positive and p16-positive status, were more prevalent in the RS group. One potential explanation for the result is the better response to radiation therapy in HPV-positive or p16-positive tumors. This finding is consistent with the increased radiosensitivity reported by other groups [7, 16]. A recent study [26] of 26 HNSCC cases using flow cytometry analysis demonstrated that PD-1-positive TIL was increased in HPV-positive patients. In our large population, we demonstrated 
that PD-1 expression levels are significantly increased in the HPV-positive group and p16-positive group. A recent study [11] suggested that high mutation load correlates with improved response to anti-PD1 immunotherapy. The difference in mutation burden between HPV-positive and HPV-negative tumors may lead to the difference in PD-L1 expression. Thus, we hypothesized that HPV-mediated PD-1 expression could increase radiosensitivity. This hypothesis should be investigated in further studies.

We summarized recent studies concerning PD-L1 and PD-1 in HNCs in Table S4. In published correlative studies [17, 21], high PD-L1 expression was associated with worse OS and an increased risk for nodal metastasis. Lin, Y. M. et al. [20] reported high PD-L1 expression as a risk factor for OS in males and smokers, which was consistent with us. Our results indicate that PD-L1 expression was a negative prognostic factor for head and neck cancer in patients expressing the radioresistant gene signature, but the underlying mechanism is unclear. We previously demonstrated that EGFR-mediated endoplasmic reticulum stress (ERS) induced radioresistance in oropharyngeal carcinoma $[27,28]$ and was confirmed in this study. Concha-Benavente, F. et al. [24] reported that PD-L1 expression was induced in an EGFR-dependent manner. Thus, this phenomenon could be explained by the fact that PD-L1 upregulation is caused by EGFR, which inhibits the activation of CD8-positive T cells and causes tumor immune escape [29]. In contrast, our results are not consistent with previous studies demonstrating an adverse prognostic effect of PD-L1 expression in several subgroups of HNCs, including laryngeal cancer, oropharynx cancer and HPV-negative tumors $[19,30]$. Vassilakopoulou, M. et al. [30] suggested that partial antitumor immunity may explain the observed survival benefit. Furthermore, in their study, automated quantitative protein analysis (AQUA) was employed to measure PD-L1 protein levels. This technique differs from immunohistochemistry employed in the majority of studies. In addition, the number of cases should also be considered. We also observed a significant positive prognostic value of high PD-1 expression. Recent studies indicate that low PD-1 expression was associated with increased risk for local recurrence and reduced OS [7, 22], which were similar with our findings. Interestingly, Kansy, B. A. et al. [31] reported that cells with extremely high PD-1 expression indicated significantly worse disease-free survival and an increased risk for recurrence. These findings warrant further studies.

Some limitations should be taken into account. First, immunohistochemistry was frequently adopted to measure gene expression in previous studies.
However, we employed mRNA expression level as a surrogate. Second, the threshold of PD-L1 expression to determine high or low expression was dependent on the researchers' subjectivity. In this study, our classification was based on the 25th percentile and 75th percentile of transformed RSEM values of mRNA level. This method was adopted from previous reports $[15,32]$.

Our innovation in this research is the value of basal PD-1/PD-L1 expression in predicting the radiosensitivity and prognostic role in the context of radiosensitivity and HPV status. Previously, animal studies were used to investigate combination strategies using blockade of PD-L1 or PD-1 with radiation in different tumor types to overcome radioresistance $[33,34]$. The changes in PD-L1 expression due to radiotherapy were the main targets of previous studies [35]. However, the basal and intrinsic PD-1 and PD-L1 expression before radiation may predict the response to radiation therapy and was rarely reported. Recently, Fiedler, M. et al. [7] suggested that basal PD-L1 expression indicated radiosensitivity but failed to reach statistical significance. Numerous factors could explain this finding, such as the number of cases, stages of cancer involved, and PD-1 and PD-L1 evaluation methods. In this study, we first demonstrate that high basal PD-1 and PD-L1 expressions are strongly related to radiosensitivity in head and neck cancer.

Unfortunately, patients with HPV-negative tumors still have a high risk of locoregional failure and low 3-year survival rates and require more efficacious treatments. In the past few decades, the 5 -year survival rate of radical radiotherapy has remained low. Radioresistant patients require a combination treatment. However, the prognostic roles of PD-1 and PD-L1 are still controversial (Table S4). Our results first indicated that PD-L1 expression was a negative prognostic factor for head and neck cancer in $\mathrm{HPV} / \mathrm{p} 16$-negative patients expressing the radioresistant gene signature. This result indicates that patients with the radioresistant gene signature and HPV/p16-negative status seem to benefit more from radiotherapy combined with anti-PD-1/PD-L1 therapy. These findings provide insight into radioresistant and HPV-negative tumor treatment, particularly with respect to clinical trials in the future.

\section{Supplementary Material}

Supplementary figures and tables. http://www.jcancer.org/v10p0937s1.pdf

\section{Acknowledgements}

This work is supported by Project 81402521 by the National Natural Science Foundation of China. 


\section{Ethics}

The study was approved by the Ethics Committee of the First Hospital of China Medical University, Shenyang, China.

\section{Competing Interests}

The authors have declared that no competing interest exists.

\section{References}

1. Ferlay J, Soerjomataram I, Dikshit R, Eser S, Mathers C, Rebelo M, et al. Cancer incidence and mortality worldwide: sources, methods and major patterns in GLOBOCAN 2012. International journal of cancer. 2015; 136: E359-86.

2. Seiwert TY, Burtness B, Mehra R, Weiss J, Berger R, Eder JP, et al. Safety and clinical activity of pembrolizumab for treatment of recurrent or metastatic squamous cell carcinoma of the head and neck (KEYNOTE-012): an open-label, multicentre, phase $1 \mathrm{~b}$ trial. The Lancet Oncology. 2016; 17: 956-65.

3. Larkins E, Blumenthal GM, Yuan W, He K, Sridhara R, Subramaniam S, et al. FDA Approval Summary: Pembrolizumab for the Treatment of Recurrent or Metastatic Head and Neck Squamous Cell Carcinoma with Disease Progression on or After Platinum-Containing Chemotherapy. The oncologist. 2017; 22: 873-8.

4. Lhuillier C, Vanpouille-Box C, Galluzzi L, Formenti SC, Demaria S. Emerging biomarkers for the combination of radiotherapy and immune checkpoint blockers. Seminars in cancer biology. 2018; 52: 125-34

5. Spiotto M, Fu YX, Weichselbaum RR. The intersection of radiotherapy and immunotherapy: mechanisms and clinical implications. Science immunology. 2016; 1.

6. Sharabi AB, Nirschl CJ, Kochel CM, Nirschl TR, Francica BJ, Velarde E, et al. Stereotactic Radiation Therapy Augments Antigen-Specific PD-1-Mediated Antitumor Immune Responses via Cross-Presentation of Tumor Antigen. Cancer immunology research. 2015; 3: 345-55.

7. Fiedler M, Weber F, Hautmann MG, Haubner F, Reichert TE, Klingelhoffer C, et al. Biological predictors of radiosensitivity in head and neck squamous cell carcinoma. Clinical oral investigations. 2018; 22: 189-200.

8. Chaturvedi AK, Engels EA, Pfeiffer RM, Hernandez BY, Xiao W, Kim E, et al. Human papillomavirus and rising oropharyngeal cancer incidence in the United States. Journal of clinical oncology : official journal of the American Society of Clinical Oncology. 2011; 29: 4294-301.

9. Tuttle S, Hertan L, Daurio N, Porter S, Kaushick C, Li D, et al. The chemopreventive and clinically used agent curcumin sensitizes HPV (-) but not HPV (+) HNSCC to ionizing radiation, in vitro and in a mouse orthotopic model. Cancer biology \& therapy. 2012; 13: 575-84.

10. Kim HS, Kim SC, Kim SJ, Park CH, Jeung HC, Kim YB, et al. Identification of a radiosensitivity signature using integrative metaanalysis of published microarray data for NCI-60 cancer cells. BMC genomics. 2012; 13: 348

11. Li T, Zhang G, Wu P, Duan L, Li G, Liu Q, et al. Dissection of Myogenic Differentiation Signatures in Chickens by RNA-Seq Analysis. Genes. 2018; 9 .

12. Kwon MJ, Rho YS, Nam ES, Cho SJ, Park HR, Min SK, et al. Clinical implication of programmed death-ligand 1 expression in tonsillar squamous cell carcinoma in association with intratumoral heterogeneity, human papillomavirus, and epithelial-to-mesenchymal transition. Human pathology. 2018; 80: 28-39.

13. Axelsson L, Nyman J, Haugen-Cange H, Bove M, Johansson L, De Lara S, et al. Prognostic factors for head and neck cancer of unknown primary including the impact of human papilloma virus infection. Journal of otolaryngology head \& neck surgery = Le Journal d'oto-rhino-laryngologie et de chirurgie cervico-faciale. 2017; 46: 45 .

14. Jiang Y, Han Y, Sun C, Han C, Han N, Zhi W, et al. Rab23 is overexpressed in human bladder cancer and promotes cancer cell proliferation and invasion. Tumour biology : the journal of the International Society for Oncodevelopmental Biology and Medicine. 2016; 37: 8131-8.

15. Rubicz R, Zhao S, April C, Wright JL, Kolb S, Coleman I, et al. Expression of cell cycle-regulated genes and prostate cancer prognosis in a population-based cohort. Prostate. 2015; 75: 1354-62.

16. Skinner HD, Giri U, Yang L, Woo SH, Story MD, Pickering CR, et al Proteomic Profiling Identifies PTK2/FAK as a Driver of Radioresistance in HPV-negative Head and Neck Cancer. Clinical cancer research : an official journal of the American Association for Cancer Research. 2016; 22: 4643-50.

17. Garcia-Pedrero JM, Martinez-Camblor P, Diaz-Coto S, Munguia-Calzada P, Vallina-Alvarez A, Vazquez-Lopez F, et al. Tumor programmed cell death ligand 1 expression correlates with nodal metastasis in patients with cutaneous squamous cell carcinoma of the head and neck. Journal of the American Academy of Dermatology. 2017; 77: 527-33.

18. Nijkamp MM, Span PN, Bussink J, Kaanders JH. Interaction of EGFR with the tumour microenvironment: implications for radiation treatment. Radiotherapy and oncology : journal of the European Society for Therapeutic Radiology and Oncology. 2013; 108: 17-23
19. Birtalan E, Danos K, Gurbi B, Brauswetter D, Halasz J, Kalocsane Piurko V, et al. Expression of PD-L1 on Immune Cells Shows Better Prognosis in Laryngeal, Oropharygeal, and Hypopharyngeal Cancer. Applied immunohistochemistry \& molecular morphology : AIMM. 2017; 26: e79-e85.

20. Lin YM, Sung WW, Hsieh MJ, Tsai SC, Lai HW, Yang SM, et al. High PD-L1 Expression Correlates with Metastasis and Poor Prognosis in Oral Squamous Cell Carcinoma. PloS one. 2015; 10: e0142656.

21. Muller T, Braun M, Dietrich D, Aktekin S, Hoft S, Kristiansen G, et al. PD-L1: a novel prognostic biomarker in head and neck squamous cell carcinoma. Oncotarget. 2017; 8: 52889-900.

22. Balermpas P, Rodel F, Krause M, Linge A, Lohaus F, Baumann M, et al. The PD-1/PD-L1 axis and human papilloma virus in patients with head and neck cancer after adjuvant chemoradiotherapy: A multicentre study of the German Cancer Consortium Radiation Oncology Group (DKTK-ROG). Int J Cancer. 2017; 141: 594-603.

23. Bardhan K, Anagnostou T, Boussiotis VA. The PD1:PD-L1/2 Pathway from Discovery to Clinical Implementation. Frontiers in immunology. 2016; 7: 550

24. Jang BS, Kim IA. A radiosensitivity gene signature and PD-L1 status predict clinical outcome of patients with invasive breast carcinoma in The Cancer Genome Atlas (TCGA) dataset. Radiotherapy and oncology : journal of the European Society for Therapeutic Radiology and Oncology. 2017; 124: 403-10.

25. Meng J, Li P, Zhang Q, Yang Z, Fu S. A radiosensitivity gene signature in predicting glioma prognostic via EMT pathway. Oncotarget. 2014; 5: 4683-93.

26. Hong AM, Vilain RE, Romanes S, Yang J, Smith E, Jones D, et al. PD-L1 expression in tonsillar cancer is associated with human papillomavirus positivity and improved survival: implications for anti-PD1 clinical trials. Oncotarget. 2016; 7: 77010-20.

27. Qiao O, Sun C, Han C, Han N, Zhang M, Li G. Endoplasmic reticulum stress pathway PERK-eIF2alpha confers radioresistance in oropharyngeal carcinoma by activating NF-kappaB. Cancer science. 2017; 108: 1421-31.

28. Sun C, Han C, Jiang Y, Han N, Zhang M, Li G, et al. Inhibition of GRP78 abrogates radioresistance in oropharyngeal carcinoma cells after EGFR inhibition by cetuximab. PloS one. 2017; 12: e0188932.

29. Concha-Benavente F, Srivastava RM, Trivedi S, Lei Y, Chandran U, Seethala $R R$, et al. Identification of the Cell-Intrinsic and -Extrinsic Pathways Downstream of EGFR and IFNgamma That Induce PD-L1 Expression in Head and Neck Cancer. Cancer research. 2016; 76: 1031-43.

30. Vassilakopoulou $\mathrm{M}$, Avgeris $\mathrm{M}$, Velcheti V, Kotoula V, Rampias $\mathrm{T}$, Chatzopoulos K, et al. Evaluation of PD-L1 Expression and Associated Tumor-Infiltrating Lymphocytes in Laryngeal Squamous Cell Carcinoma. Clinical cancer research : an official journal of the American Association for Cancer Research. 2016; 22: 704-13.

31. Kansy BA, Concha-Benavente F, Srivastava RM, Jie HB, Shayan G, Lei Y, et al. PD-1 Status in CD8(+) T Cells Associates with Survival and Anti-PD-1 Therapeutic Outcomes in Head and Neck Cancer. Cancer research. 2017; 77: 6353-64.

32. Spahillari A, Mukamal KJ, DeFilippi C, Kizer JR, Gottdiener JS, Djousse L, et al. The association of lean and fat mass with all-cause mortality in older adults: The Cardiovascular Health Study. Nutrition, metabolism, and cardiovascular diseases : NMCD. 2016; 26: 1039-47.

33. Dovedi SJ, Adlard AL, Lipowska-Bhalla G, McKenna C, Jones S, Cheadle EJ, et al. Acquired resistance to fractionated radiotherapy can be overcome by concurrent PD-L1 blockade. Cancer research. 2014; 74: 5458-68

34. Zeng J, See AP, Phallen J, Jackson CM, Belcaid Z, Ruzevick J, et al. Anti-PD-1 blockade and stereotactic radiation produce long-term survival in mice with intracranial gliomas. International journal of radiation oncology, biology, physics. 2013; 86: 343-9.

35. Skinner HD, Giri U, Yang LP, Kumar M, Liu Y, Story MD, et al. Integrative Analysis Identifies a Novel AXL-PI3 Kinase-PD-L1 Signaling Axis Associated with Radiation Resistance in Head and Neck Cancer. Clinical cancer research: an official journal of the American Association for Cancer Research. 2017; 23: 2713-22 